\title{
Fatal H1N1 Influenza A (2009) Infection: Stroll Down the Memory Lane
}

\author{
10.5005/jp-journals-10028-1414 \\ : Prof. Ritambhra Nada ${ }^{1}$ \\ Pathology Consultant \\ Pathologist \\ Clinical Discussant \\ Radiology Discussant \\ Clinician in Charge \\ CPC Chairperson \\ Date of CPC
}

\section{Case Description}

A 24-year-old primigravida presented with fever for 4 days, diarrhea, and cough with shortness of breath for 2 days. Fever was intermittent and associated with chills and rigor. Diarrhea was watery in consistency with a frequency of 3-5 stools/day. Shortness of breath progressed gradually. There was a past history of fever associated with sore throat and arthritis at 15 years of age. However, there was no history of heart disease nor was she was taking any regular medications or penicillin prophylaxis. One week before admission, she had contact with her nephew who had a flu-like illness. Her nephew recovered in a couple of days with local medication.

\section{EXAMINATION}

She was conscious, oriented but had a toxic look. She was afebrile, had tachycardia with a pulse rate of 125 /minutes, which was regular, and there was no brachiofemoral delay. Her blood pressure was $110 / 60 \mathrm{~mm} \mathrm{Hg}$ in the right arm supine position. She was in respiratory failure with tachypnea and a respiratory rate of 32 /minutes with accessory muscle use and central cyanosis. She was pale and one of the residents had noticed raised jugular venous pressure (JVP) but no definite waveforms were appreciated. On respiratory system examination, she had bilateral extensive crepitations and bronchial breath sounds in the right mammary/axillary/infra-axillary/infraand interscapular areas. On cardiovascular examination, her S1/S2 was audible. The apex was localized to the 6th intercostal space and a mid-diastolic murmur was appreciated at the apex. Central nervous system (CNS) examination was normal. The gravid uterus was felt on the abdomen palpation. No organomegaly was noted.

\section{INVESTIGATIONS}

Ultrasound (USG) chest/abdomen showed minimal right-sided pleural effusion with underlying consolidation and mild pericardial effusion. Later USG showed a dead fetus. A compression ultrasound revealed no deep vein thrombosis. Bedside echocardiography had a normal left ventricle (LV), enlarged left atrium (LA) [left atrium inner diameter (LAID) $-50 \mathrm{~m}$ ] but no thrombus was seen. The anterior and posterior mitral leaflets were thickened. There was moderate mitral stenosis [mitral valve atrium (MVA) $1.4 \mathrm{~cm}^{2}$ ] with

\footnotetext{
${ }^{1,2}$ Department of Histopathology, Postgraduate Institute of Medical Education and Research, Chandigarh, India

${ }^{3}$ Postgraduate Institute of Medical Education and Research, Chandigarh, India

${ }^{4,7}$ Department of Medicine, Postgraduate Institute of Medical Education and Research, Chandigarh, India

${ }^{5}$ Department of Radiology, Postgraduate Institute of Medical Education and Research, Chandigarh, India

${ }^{6}$ Department of Pulmonary Medicine, Postgraduate Institute of Medical Education and Research, Chandigarh, India
}

Corresponding Author: Ritambhra Nada, Department of Histopathology, Postgraduate Institute of Medical Education and Research, Chandigarh, India, Phone: +91 172-2755143, e-mail: ritamduseja@yahoo.com

How to cite this article: Nada R, Bal A, Thirunavukkarasu B, et al. Fatal H1N1 Influenza A (2009) Infection: Stroll Down the Memory Lane. J Postgrad Med Edu Res 2021;55(2):101-105.

Source of support: Nil

Conflict of interest: None

mild tricuspid regurgitation. No vegetations were noted on the valves. The X-ray chest pulmonary artey $(\mathrm{PA})$ view showed bilateral (right $>$ left) patchy consolidation and nodular opacities, coalescing at places and had a predominant central, perihilar distribution with relative sparing of lung periphery and lung ape. Right CP angle was blunt, suggestive of pleural effusion. There was cardiomegaly with straightening of left heart border, suggestive of mitral valve disease. Electrocardiogram (ECG) showed sinus tachycardia and left atrial enlargement. Antinuclear antibody (ANA) was negative and urine routine examination showed: Albumin,$++ 8-10$ red blood cells/ high power field (RBC/HPF), and rare pus cells. Blood culture and endotracheal (ET) aspirate cultures were sterile. Throat/nasal swab for H1N1 by real-time (RT) PCR was positive. Refer to Tables 1 and 2 for other investigations (Figs 1 and 2).

\section{Course and Management}

This 24-year-old pregnant woman ( 18 weeks) presented to a medical emergency with a short febrile illness followed by progressive 
dyspnea of recent onset, associated with a mild cough and streaky hemoptysis. She was hypoxic at admission, which was corrected with high flow oxygen; soon requiring noninvasive ventilation, later assisted mechanical ventilation. With a high possibility of an H1N1 2009 infection, she was given a double dose (150 mg BD) of oseltamivir (Tamiflu). Echocardiography confirmed the diagnosis of mitral stenosis and she initially received decongestive therapy in the form of diuretics and nitroglycerine (NTG) infusion. Intravenous (IV) antimicrobials (ceftriaxone and azithromycin) were given on day 1 of admission, which was later, changed to piperacillin-tazobactam and vancomycin on day 3 of admission. On day 2, she suffered an episode of bradycardia with hypotension, revived after atropine, and chest compressions. Following this event, she received central venous pressure (CVP)-guided fluids, multiple inotropes, and hydrocortisone for refractory hypotension. Preterminally, acute right upper limb ischemia was noticed in the form of absent pulses. Her renal functions rapidly declined but renal replacement (RRT) could not be given. On day 4 of hospital stay, she suffered a cardiorespiratory arrest from which she could not be revived.

\section{Case Analysis}

This is a 24-year-old pregnant woman with a suspected past history of acute rheumatic fever presenting with fever, diarrhea, and acute onset respiratory distress. The fever was initially mild which persisted with a rising trend and had leukocytopenia

Table 1: Investigation

\begin{tabular}{|c|c|c|c|}
\hline Date & 21.12 .09 & 22.12 .09 & 23.12 .09 \\
\hline $\mathrm{Hb}(\mathrm{g} / \mathrm{dL})$ & 11.2 & 8.9 & \\
\hline $\mathrm{TLC} / \mathrm{mm}^{3}$ & 2,300 & & \\
\hline DLC & P40/L9/M1 & & \\
\hline Platelets $/ \mathrm{mm}^{3}$ & 35,000 & 45,000 & \\
\hline $\mathrm{Na}+(\mathrm{mEq} / \mathrm{L})$ & 138 & 145 & 143 \\
\hline $\mathrm{K}+(\mathrm{mEq} / \mathrm{L})$ & 4.6 & 5.17 & \\
\hline Urea (mg/dL) & 38 & 80.2 & 138.5 \\
\hline $\begin{array}{l}\text { Creatinine (mg/ } \\
\mathrm{dL} \text { ) }\end{array}$ & 0.5 & 1.87 & 4.87 \\
\hline Bilirubin T/C & 1.10 & & \\
\hline S. protein T/A & $7.26 / 3.8$ & & \\
\hline SGOT & 75.3 & & \\
\hline SGPT & 23.74 & & \\
\hline Alk $\mathrm{PO}_{4}(\mathrm{U} / \mathrm{L})$ & 90 & & \\
\hline $\mathrm{Ca}+(\mathrm{mg} / \mathrm{dL})$ & 8.0 & & \\
\hline $\begin{array}{l}\text { Phosphate } \\
\text { (mg/dL) }\end{array}$ & 5.3 & & \\
\hline PT & 10 & 17 & \\
\hline PTI & 100 & 59 & \\
\hline aPTT & 54 & 54 & \\
\hline
\end{tabular}

Bold are abnormal values suggesting a viral origin. She also had a contact history. On investigation, there were extensive nodular opacities in the lungs with positive RT-PCR for H1N1 (2009) indicting acute respiratory distress syndrome (ARDS) due to influenza. The causes of ARDS in pregnancy can be multifactorial and can be classified based on its relation to pregnancy (Table 3). ${ }^{1-3}$ Leading cause of acute respiratory distress in pregnancy was an infection, followed by preeclampsia and aspiration. ${ }^{3}$ Pregnant women are at increased risk of morbidity and mortality during the H1N1 pandemic during 2009 both in India and globally. ${ }^{4-6}$ Role of pregnancy in respiratory illness has been attributed to altered pulmonary mechanics and suppressed cell and humoral immunity. ${ }^{7}$ This patient had a contact with cousin with a similar illness. The household secondary attack rate of H1N1 2009 was estimated to be about $27.3 \% .^{8}$ There is a possibility of bacterial/fungal coinfection in this patient as there was multifocal consolidation, and high-grade fever. Multiple autopsy series provide evidence for such association. ${ }^{7}$ Common viral etiological agents include influenza $A$, influenza $B$, and varicellazoster virus while common bacterial agents are Streptococcus pneumoniae, Haemophilus influenzae, Mycoplasma pneumoniae, etc. ${ }^{9}$ A public health surveillance study reported a case fatality rate of $22(9-56)$ deaths per 100,000 and two-thirds had a severe systemic disease or incapacitating systemic illness. ${ }^{10}$ In addition to pregnancy, this patient had an increased risk of pneumonia due to underlying rheumatic mitral valve disease. ${ }^{8,11}$ Additional high-risk factors associated in this case are coinfection (bacterial/ fungal), non-vaccination, and admission into intensive care. ${ }^{9}$ Terminal complications included anemia, thrombocytopenia, and hypotension probably due to associated disseminated intravascular coagulation, infection-related hemophagocytosis, and septic shock.

\section{Final Clinical Diagnosis}

- Primigravida with intrauterine fetal death.

- Moderate mitral stenosis (etiology probably rheumatic), normal sinus rhythm, no evidence of infective endocarditis.

- H1N1 influenza (2009) pneumonia, ARDS, sepsis.

- Refractory septic shock, acute kidney injury.

\section{Pathology Findings}

A partial autopsy was performed. Prosectors noted that the deceased was moderately built with pedal edema. Each pleural cavity yielded $200 \mathrm{~mL}$ of serous fluid, pericardial cavity yielded 150 $\mathrm{mL}$ of serous fluid. The peritoneal cavity was within normal limits.

Both the lungs together weighed 1,600 g. They were heavy, airless, and subcrepitant to feel. Pleurae were dull and showed subpleural hemorrhages. On the cut surface, both the lungs showed diffuse hemorrhagic consolidation with obliteration of the lung markings giving a homogeneous appearance. Focal nodular hemorrhagic lesions were palpable. Main pulmonary vessels were within normal limits, no thrombi were seen on gross.

Table 2: ABG analysis

\begin{tabular}{llllllll}
\hline & $\mathrm{pH}$ & $\mathrm{PaO}_{2}$ & $\mathrm{PaCO}_{2}$ & $\mathrm{HCO}_{3}$ & $\mathrm{BE} / \mathrm{BD}$ & $\mathrm{SPO}_{2} \%$ & $\mathrm{FiO}$ \\
\hline 21.12 .09 & 7.415 & $\mathbf{3 6 . 3}$ & 31.4 & 19.7 & -3.7 & $\mathbf{6 9 . 7}$ & $0.45(\mathrm{Venturi})$ \\
21.12 .09 & 7.431 & $\mathbf{4 8 . 6}$ & 25.9 & 16.9 & -5.6 & $\mathbf{8 4 . 6}$ & $\mathrm{NIV}\left(\mathrm{O}_{2} 15 \mathrm{~L} / \mathrm{mt}\right)$ \\
21.12 .09 & 7.178 & 71.9 & 71.7 & 25.8 & -4.3 & $\mathbf{8 8 . 5}$ & $\mathrm{MV}\left(100 \% \mathrm{O}_{2}\right)$ \\
22.12 .09 & 7.248 & 91.8 & 48.5 & 20.7 & -6.7 & $\mathbf{9 5 . 1}$ & $\mathrm{MV}\left(100 \% \mathrm{O}_{2}\right)$ \\
23.12 .09 & 7.038 & $\mathbf{7 1 . 8}$ & $\mathbf{5 9 . 5}$ & 15.6 & -15.6 & $\mathbf{8 1 . 7}$ & ${\mathrm{MV}\left(100 \% \mathrm{O}_{2}\right)}$ \\
\hline
\end{tabular}



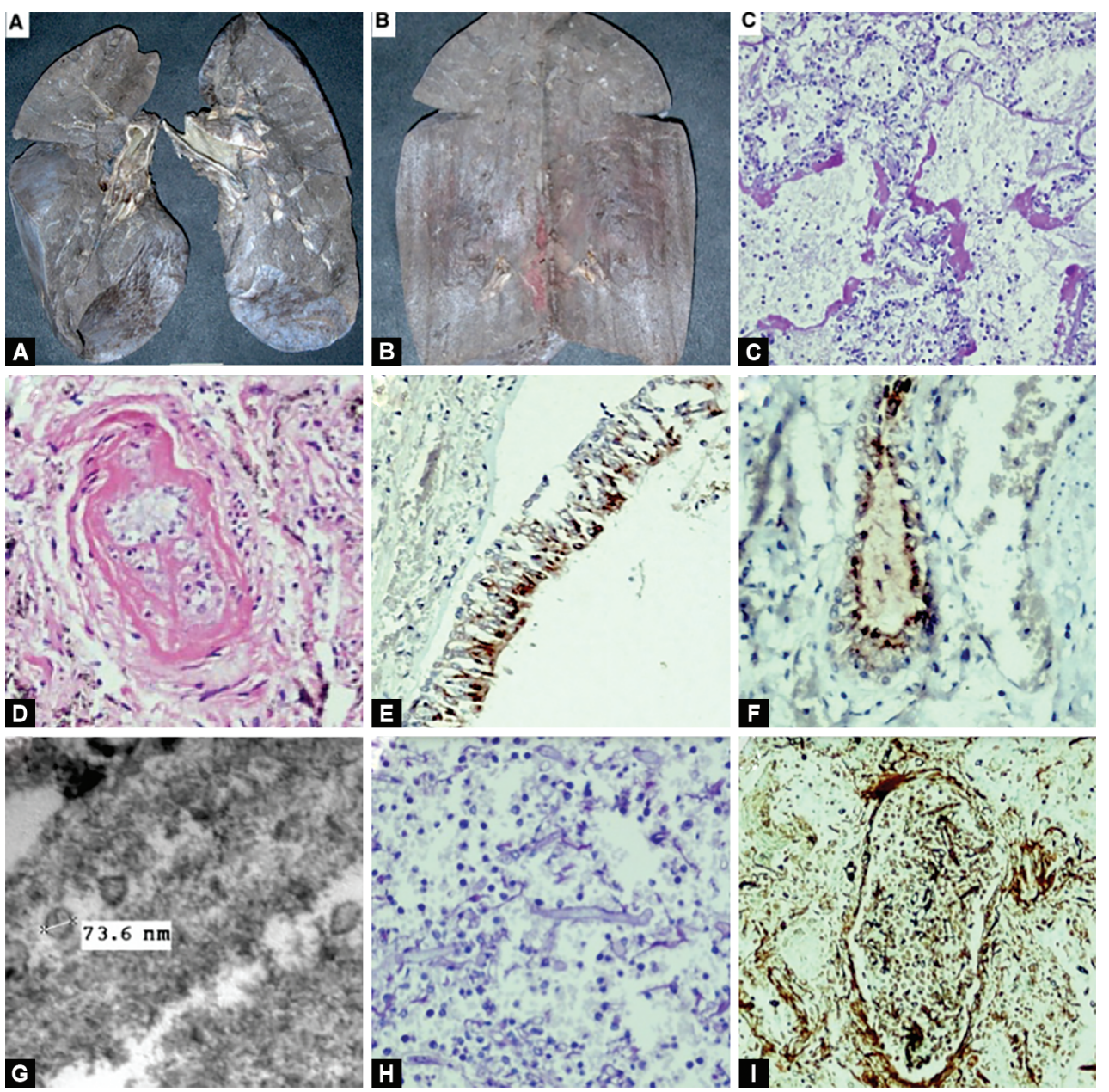

Figs 1 A to I: (A) Both the lungs were heavy; (B) The cut surface shows hemorrhagic nodular opacity; (C) PAS-positive hyaline membrane deposition in alveolar ducts; (D) Fibrin thrombi with endothelial injury; ( $E$ and F) $\mathrm{H} 1 \mathrm{~N} 1$ antibody positive in the columnar epithelium and distal bronchi (DAB; 40X); (G) Viral particles ranging in size from 74 to $82 \mathrm{~nm}$ in diameter with spikes of surface glycoproteins on the outer surface, characteristics of the influenza virus; (H) Broad, pauci-septate hyphae of zygomycosis; (I) Grocott's Methenamine Silver stain highlighting angioinvasive fungal profiles

A microscopic examination from the representative section showed the desquamation of bronchial lining with a moderate lymphomononuclear infiltrate in the wall and submucosal glands along with congestion. Lung parenchyma showed widespread fibrin deposition lining the alveolar ducts and spaces leading to the formation of a hyaline membrane. There was interstitial edema, congested capillaries, and mild lymphomononuclear cell infiltrate. Interstitial small and medium blood vessels showed fibrin thrombi with injury to lining endothelial cells indicating microangiopathy. There was a shedding of bronchiolar lining with similar hyaline material lining the bronchiolar wall indicating fibrinous bronchiolitis. Sections from the nodular consolidated area show alveolar hemorrhage with many broad, pauci-septate, angioinvasive fungal hyphae with morphological features of zygomycosis.

\section{Immunohistochemistry (Anti-influenza A H1N1 Antibody)}

Showed nuclear and cytoplasmic positivity in bronchial and bronchiolar lining epithelium, submucosal glands, and gland ducts. Occasional alveolar macrophage also showed cytoplasmic positivity. However, type I and type II pneumocytes were negative.

\section{On Electron Microscopy from Tracheal Ciliated Columnar Epithelium}

Viral particles were observed. Each viral particle ranged in size from 74 to $82 \mathrm{~nm}$ in diameter. In some particles, the spikes of surface glycoproteins on the outer surface, characteristics of the influenza virus were seen. Real-time PCR for H1N1 was positive in the lung, while negative in the heart, liver, and placenta.

The heart weighed $400 \mathrm{~g}$. Epicardial hemorrhages were seen. The left atrium was dilated and the mitral valve ring diameter was reduced. The mitral leaflets were diffusely thickened with commissural fusion and shortened and thickened chordae tendineae. Aortic valve cusps were also thickened with commissural fusion. There was dilatation of the root of the aorta. Tricuspid and pulmonary valves were within normal limits. Left ventricular wall thickness was $1.5 \mathrm{~cm}$ and right ventricular wall thickness was 0.4 $\mathrm{cm}$. The microscopic section from the mitral valve and aortic valve leaflet showed dense fibrocollagenous tissue with prominent thick-walled blood vessels and focal inflammatory cell infiltrate comprising of lymphocytes and histiocytes indicating chronic valvulitis.

The liver weighed $990 \mathrm{~g}$. The capsular surface was normal. The cut surface showed exaggerated mottling at places becoming 

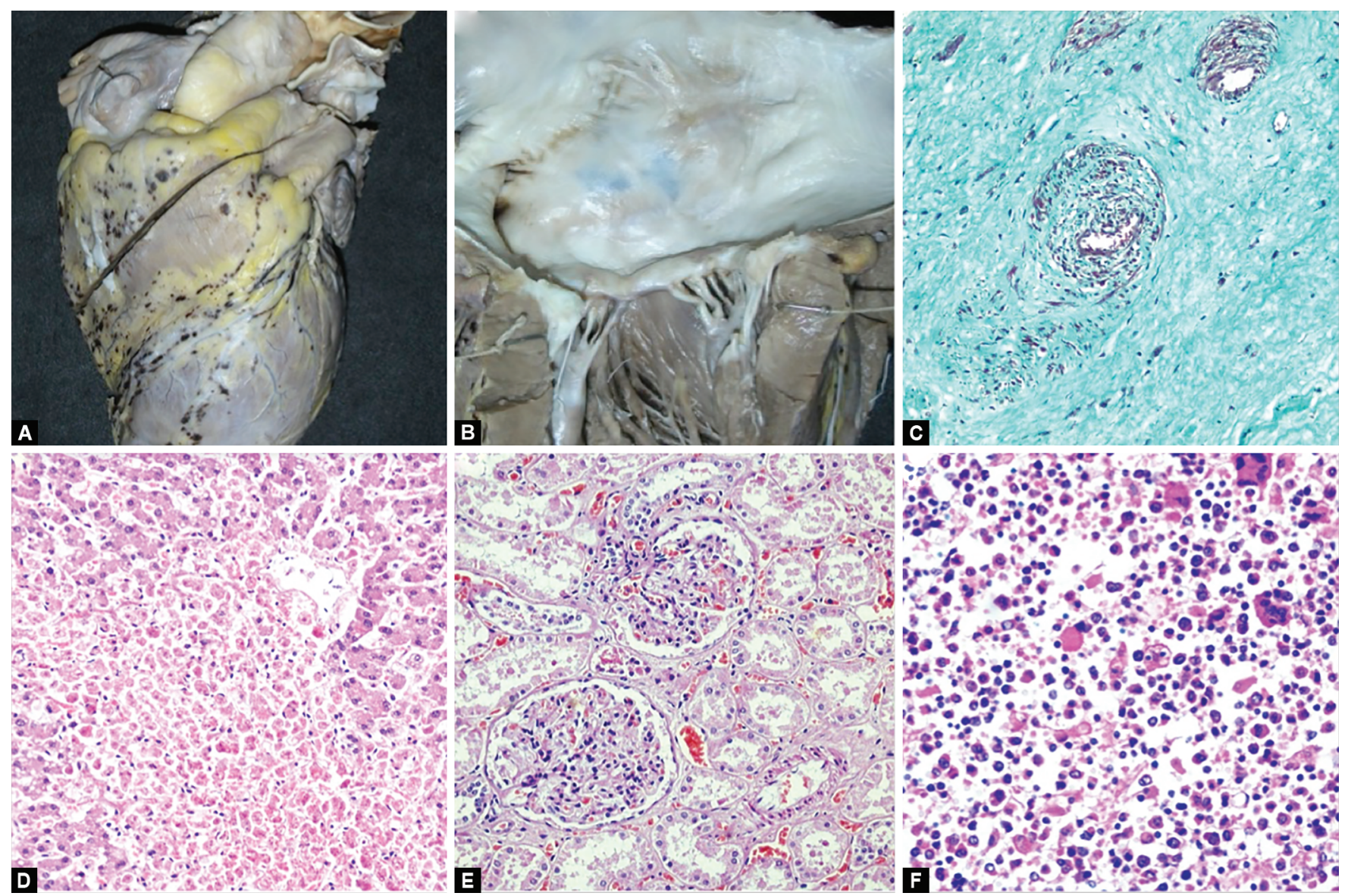

Figs 2A to F: (A) Epicardial hemorrhages; (B) Left atrial dilatation with thickened mitral valve with fused commissures; (C) Masson trichrome stained valve highlights fibrosis and neovascularization; (D) Centrizonal hemorrhagic necrosis of liver; (E) Acute tubular necrosis of kidney; (F) Bone marrow section showing erythrophagocytosis ( $\mathrm{H} \& \mathrm{E} ; 40 \mathrm{X})$

Table 3: Causes of acute respiratory distress syndrome during pregnancy

\begin{tabular}{|c|c|}
\hline Unique to pregnancy & Not unique to pregnancy \\
\hline Hypertensive disorder & Venous thromboembolism \\
\hline Amniotic fluid embolism & Cardiovascular disease \\
\hline $\begin{array}{l}\text { Ovarian hyperstimulation } \\
\text { syndrome }\end{array}$ & Pulmonary artery hypertension \\
\hline Acute fatty liver of pregnancy & Bronchial asthma \\
\hline Peripartum cardiomyopathy & Pneumonia \\
\hline Chemical pneumonitis & Neuromuscular disorders \\
\hline $\begin{array}{l}\text { Tocolytic-induced pulmonary } \\
\text { edema }\end{array}$ & Sepsis \\
\hline $\begin{array}{l}\text { Septic abortion, chorioamnioni- } \\
\text { tis, endometritis }\end{array}$ & Fat embolism, drug overdose \\
\hline
\end{tabular}

confluent. The portal vein and inferior vena cava were within normal limits. No thrombus was seen. The extrahepatic biliary tree was within normal limits. Representative sections from the liver showed extensive centrizonal hemorrhagic necrosis of hepatocytes and sinusoidal dilatation. The portal tracts were within normal limits.

The spleen weighed $250 \mathrm{~g}$. It was mildly enlarged and congested. Microscopy showed red pulp congestion.

Both the kidneys together weighed $260 \mathrm{~g}$. The external surface appeared swollen with a blotchy appearance; the cut surface showed marked medullary congestion. Microscopy showed normal glomeruli with features of acute tubular necrosis.

The pancreas was grossly and microscopically unremarkable.

Sections from the stomach and small intestine showed mild mucosal ischemia.

The uterus was bulky and gravid. The placenta showed interand perivillous villous fibrin deposition. Section form lymph nodes and bone marrow showed hemophagocytosis.

\section{Final Autopsy Diagnosis}

A 24-year-old primigravida with intrauterine fetal death:

- Diffuse alveolar damage, bronchitis, and necrotizing bronchiolitis (influenza A H1N1 associated).

- Invasive pulmonary zygomycosis.

- Chronic rheumatic valvulitis (mitral and aortic stenosis).

- Hypoxic changes in:

- Liver-Centrizonal hemorrhagic necrosis.

- Acute tubular necrosis.

- Mucosal ischemia in gastrointestinal tract.

- Placenta.

- Hemophagocytosis: Lymph nodes, bone marrow.

\section{Discussion}

This case demonstrates clinical, pathological, and virological findings in a fatal H1N1 infection. The target group to develop 
severe disease includes people with a prior lack of exposure to illness, obesity, pregnancy, and cardiovascular diseases. There are no definitive explanations for increased risk of viral pneumonia in pregnancy and patients with heart disease. The plausible hypothesis includes pulmonary hypertension in cardiac diseases and increased blood volume in pregnancy predisposing to alveolar edema and septal damage by the virus.

The histological findings of acute alveolar injury in the form of diffuse alveolar damage caused to be seen in many other viral infections including coronavirus and other agents that are injurious to alveoli. The alveoli and alveolar ducts are lined by hyaline membranes, consisting of fibrin mixed with remnants of necrotic epithelial cells. Fibrin thrombi were present in the capillaries of alveolar septa and alveolar ducts, as well as in small pulmonary blood vessels. Immunohistochemistry demonstrated a higher viral load in the ciliated columnar epithelium of proximal airway than the alveolar cells and pneumocytes, which was further confirmed with the ultrastructural examination. The damage to distal lung parenchyma and other organs was cytokine-mediated end-organ damage rather than a direct viral cytopathic effect. This distribution of viral load is well known in the existing literature. ${ }^{7,12}$ Various autopsy series have described secondary bacterial, viral, and/or fungal coinfection in influenza A H1N1 infection depending on the host environment. ${ }^{10,13}$ This case demonstrated the coinfection of angioinvasive zygomycosis. Another interesting finding is the lack of transplacental spread of the virus. Reverse transcriptase polymerase chain reaction ( $\mathrm{RRT}-\mathrm{PCR}$ ) performed in the placenta, fetal liver, and heart was negative.

Taking note of the ongoing COVID-19 pandemic, this case demonstrates another fatal pandemic viral infection with many similar histomorphological features.

\section{References}

1. Lapinsky SE. Acute respiratory failure in pregnancy. Obstet Med 2015;8(3):126-132. DOI: 10.1177/1753495X15589223.
2. Bhatia P, Biyani G, Mohammed $S$, et al. Acute respiratory failure and mechanical ventilation in pregnant patient: a narrative review of literature. J Anaesthesiol Clin Pharmacol 2016;32(4):431. DOI: 10.4103/0970-9185.194779.

3. Catanzarite V, Willms $D$, Wong $D$, et al. Acute respiratory distress syndrome in pregnancy and the puerperium. Obstet Gynecol 2001;97(5):760-764. DOI: 10.1097/00006250-200105000-00022.

4. Malhotra B, Singh R, Sharma P, et al. Epidemiological \& clinical profile of influenza A (H1N1) 2009 virus infections during 2015 epidemic in Rajasthan. Indian J Med Res 2016;144(6):918. DOI: 10.4103/ijmr. IJMR_1183_15.

5. Singhal S, Sarda N, Arora R, et al. Clinical profile \& outcome of H1N1 infected pregnant women in a tertiary care teaching hospital of northern India. Indian J Med Res 2014;139:454-458.

6. Mosby LG, Rasmussen SA, Jamieson DJ. 2009 pandemic influenza A (H1N1) in pregnancy: a systematic review of the literature. Am J Obstet Gynecol 2011;205(1):10-18. DOI: 10.1016/j.ajog.2010. 12.033 .

7. Gill JR, Sheng Z-M, Ely SF, et al. Pulmonary pathologic findings of fatal 2009 pandemic influenza A/H1N1 viral infections. Arch Pathol Lab Med 2010;134:235-243.

8. Pande A, Saluja M, Nandwani S. Influenza a infection unmasking an underlying mitral valve stenosis in a 19-year-old boy. J Fam Med Prim Care 2013;2(2):204. DOI: 10.4103/2249-4863.117427.

9. Minchole E, Figueredo AL, Omeñaca M, et al. Seasonal influenza A H1N1pdm09 virus and severe outcomes: a reason for broader vaccination in non-elderly, at-risk people. PLoS ONE 2016;11(11):e0165711. DOI: 10.1371/journal.pone.0165711.

10. Mukhopadhyay S, Philip AT, Stoppacher R. Pathologic findings in novel influenza A (H1N1) virus ("Swine Flu") infection. Am J Clin Pathol 2010;133(3):380-387. DOI: 10.1309/AJCPXY17SULQKSWK.

11. Placzek HED, Madoff LC. Association of age and comorbidity on 2009 influenza A pandemic H1N1-related intensive care unit stay in Massachusetts. Am J Public Health 2014;104(11):e118-e125. DOI: 10.2105/AJPH.2014.302197.

12. Kuiken T, Taubenberger JK. Pathology of human influenza revisited. Vaccine 2008;26(Suppl 4):D59-D66. DOI: 10.1016/j. vaccine.2008.07.025

13. Bal A, Suri V, Mishra B, et al. Pathology and virology findings in cases of fatal influenza A H1N1 virus infection in 2009-2010. Histopathology 2012;60(2):326-335. DOI: 10.1111/j.1365-2559.2011.04081.x. 